\title{
Quantum mechanical transition state theory and tunneling corrections
}

\author{
Ward H. Thompson \\ Department of Chemistry and Biochemistry, University of Colorado, Boulder, Colorado 80309-0215
}

(Received 7 October 1998; accepted 1 December 1998)

\begin{abstract}
An efficient implementation of the quantum mechanical transition state theory recently proposed by Hansen and Andersen [J. Chem. Phys. 101, 6032 (1994); J. Phys. Chem. 100, 1137 (1996)] is presented. Their method approximates the flux-flux autocorrelation function by using short-time information to fit an assumed functional form (with physically correct properties). The approach described here exploits the low rank of the half-Boltzmannized flux operator, thereby facilitating application to reactions involving many degrees of freedom. In addition, we show how the quantum transition state theory can be used to obtain tunneling corrections within the framework of more traditional transition state theory approaches, i.e., those making an assumption of separability. Directions for possible improvements of the theory are discussed. (C) 1999 American Institute of Physics. [S0021-9606(99)02009-7]
\end{abstract}

\section{INTRODUCTION}

Classical transition state theory ${ }^{1}$ (TST) today remains as one of the most powerful techniques for computing thermal rate constants for chemical reactions, particularly for larger systems. Its usefulness is due to a combination of factors: It is easy to implement. There is a clear physical picture of the approximation invoked, i.e., the assumption that no trajectories recross the transition state. ${ }^{2}$ It provides a rigorous upper bound to the exact classical rate. And, finally, it yields accurate rate constants for systems obeying classical mechanics and exhibiting direct dynamics.

However, often one is interested in chemical reactions where classical mechanics is not a valid description, e.g., light atom $(H)$ transfer reactions which can proceed by tunneling. Thus, a quantum mechanical transition state theory with properties analogous to those listed above would be of immense value. Despite the efforts of many workers, ${ }^{3-9}$ no theory satisfying all of these requirements has been developed. One problem is that no meaningful upper bound to the exact quantum mechanical rate has been found. A larger difficulty is that of translating the fundamental assumption of classical transition state theory, "no recrossing trajectories," into a quantum mechanical framework. Because of this ambiguity many different quantum mechanical transition state theories have been proposed. While the ultimate goal of a unique quantum mechanical analogue of classical transition state theory has not been achieved, there are several quantum mechanical transition state theories which provide accurate methods for calculating thermal rate constants based on an assumption of "direct dynamics" (yielding a significant reduction in the computational effort).

Recently, Hansen and Andersen proposed a quantum mechanical transition state theory ${ }^{8,9}$ based on the flux-flux autocorrelation function which is capable of accurately representing tunneling (including nonseparability). The fluxflux autocorrelation function provides a direct route (i.e., with no reference to state-selected or energy-dependent quantities) to the exact thermal rate constant for a chemical reaction,,${ }^{10,11}$

$$
k(T)=\frac{1}{Q_{r}(T)} \int_{0}^{\infty} C_{f f}(t) d t,
$$

where $Q_{r}(T)$ is the reactant partition function per unit volume and

$$
C_{f f}(t)=\operatorname{tr}\left[e^{-\beta \hat{H} / 2} \hat{F} e^{-\beta \hat{H} / 2} e^{i \hat{H} t / \hbar} \hat{F} e^{-i \hat{H} t / \hbar}\right]
$$

is the flux-flux autocorrelation function. Here $\hat{H}$ is the Hamiltonian, $\hat{F}$ is the flux operator defined for a dividing surface separating reactants and products, and $\beta=1 / k_{b} T$ with $k_{b}$ as Boltzmann's constant. The transition state theory of Hansen and Andersen ${ }^{8,9}$ uses short time information about $C_{f f}(t)$ to obtain an approximation to the rate constant. Namely, the values of the correlation function and its second derivative at zero time are used to determine parameters in an assumed functional form (possessing the desired properties). In this paper we show how this TST can be efficiently implemented to make it applicable to large chemical systems. We also outline how it can be used to obtain a tunneling correction for more traditional (i.e., separable) TSTs. In addition, it can be utilized to improve the separability approximation in such cases by explicitly including several strongly coupled degrees of freedom.

As has been shown previously, the Boltzmannized flux operator,

$$
\hat{F}(\beta)=e^{-\beta \hat{H} / 2} \hat{F} e^{-\beta \hat{H} / 2},
$$

is of low rank (i.e., it has only a small number of nonzero eigenvalues). ${ }^{12}$ This is true because the flux operator in a single dimension has only two nonzero eigenvalues (in a finite basis representation), equal in magnitude and opposite in sign (corresponding to forward and backward flux). ${ }^{13-15} \mathrm{In}$ a multidimensional case the low rank is preserved by the Boltzmann factor which limits the contribution from the degrees of freedom parallel to the dividing surface to states of lower energy. (Naturally the number of these states increases 
with temperature.) Thus, if the dividing surface is placed at the transition state, the number of nonzero eigenvalues of $\hat{F}(\beta)$ is approximately twice the number of thermally accessible states of the activated complex at temperature $T$. This fact has previously been exploited by Miller and co-workers ${ }^{12,16-18}$ and Manthe and co-workers ${ }^{19,20}$ in the calculation of exact thermal rate constants for gas-phase chemical reactions (including recombination processes ${ }^{18,21}$ ). Significant progress in this area has also been made by Light and co-workers. ${ }^{13,14,22,23}$

Section II describes the implementation of the quantum TST of Hansen and Andersen, including how the low rank of the half-Boltzmannized flux operator can be used to advantage. An illustrative application to the $\mathrm{D}+\mathrm{H}_{2}$ reaction is discussed in Sec. III. The separable transition state theory approach is outlined in Sec. IV and the tunneling correction is derived in terms of $C_{f f}(t)$. Section $\mathrm{V}$ describes the application of the theory to a one-dimensional double well potential bilinearly coupled to a harmonic bath. The calculated rate constants are presented in Sec. V B and comparison is made to exact results. ${ }^{24}$ Finally, Sec. VI presents concluding remarks and some directions for future improvements.

\section{TRANSITION STATE THEORY APPROXIMATION}

The transition state theory of Hansen and Andersen uses the values of $C_{f f}(0)$ and $\ddot{C}_{f f}(0)$ (where each dot implies a time derivative) to determine the parameters in an assumed functional form for $C_{f f}(t)$. Specifically, they suggest two possibilities. $^{8,9}$ The first is the flux-flux autocorrelation function for the parabolic barrier ${ }^{11}$

$$
\begin{aligned}
C_{f f}^{\mathrm{pb}}(t)= & \frac{k T}{h}\left(\hbar \beta \omega_{b} / 2\right) \\
& \times \frac{\omega_{b} \sin \left(\beta \hbar \omega_{b} / 2\right) \cosh \left(\omega_{b} t\right)}{\left[\sin ^{2}\left(\beta \hbar \omega_{b} / 2\right)+\sinh ^{2}\left(\omega_{b} t\right)\right]^{3 / 2}} e^{-\beta E_{b},}
\end{aligned}
$$

where the two adjustable parameters are $\omega_{b}$, the barrier frequency, and $E_{b}$, the barrier height. The second is a form based on the Padé approximant to the function $d \ln \left[C_{f f}\left(z^{1 / 2}\right)\right] / d z$ giving the functional form for the correlation function as ${ }^{9,25}$

$$
C_{f f}^{\mathrm{PA}}(t)=\frac{a e^{b t^{2}}}{\left[t^{2}+\beta^{2} \hbar^{2} / 4\right]^{3 / 2}},
$$

with

$$
a=(\beta \hbar / 2)^{3} C_{f f}(0)
$$

and

$$
b=\frac{6}{(\beta \hbar)^{2}}+\frac{\ddot{C}_{f f}(0)}{2 C_{f f}(0)}
$$

as the adjustable parameters. Note that Eq. (2.2) has the correct properties as a function of complex time ${ }^{9}$ (i.e., it is analytic in the same regions as the true $C_{f f}(t)$ and has singularities in the proper places). Both correlation function forms have the correct short time behavior. ${ }^{26}$
In Secs. III and $\mathrm{V}$ we implement the transition state theory of Hansen and Andersen using the form for the correlation function given in Eq. (2.2). We choose this form rather than the parabolic barrier correlation function of Eq. (2.1) because it is more robust, i.e., it is not always possible to obtain the parameters $\omega_{b}$ and $E_{b} \cdot{ }^{9}$ Values for $a$ and $b$ in Eq. (2.2) can always be found but may not always be meaningful (see Sec. V), however this is reasonably rare.

The expressions in Eqs. (2.1) and (2.2) for the correlation function are positive for all times and therefore can be considered to represent direct dynamics in the spirit of transition state theory. [Note that, Eq. (1.1), negative values for the correlation function diminish the rate constant.] At the same time, this naturally limits the accuracy of the resulting rate as no negative lobe in the correlation function (due to "recrossing" of the flux dividing surface) can be reproduced. This implies the usefulness of these methods will be limited to reactions where there is not significant recrossing of the transition state (as would be expected). Using only the zeroth and second derivatives of $C_{f f}(t)$ at $t=0$, one has no choice but to choose a monotonically decaying function. That is, these quantities give information about the initial value of the correlation function and its initial rate of decay. More derivatives are necessary to obtain meaningful information about recrossing (i.e., to describe a negative lobe in the correlation function). Hansen and Andersen applied their quantum TST to the symmetric and asymmetric Eckart barriers as well as a parabolic barrier linearly coupled to a harmonic oscillator and found quite good agreement. ${ }^{8,9}$ However, the method did not always give a rate larger than the exact value and so does not represent an upper bound.

The flux-flux correlation function, Eq. (1.2), can be expressed (in a form convenient for the present purpose) as ${ }^{14,22}$

$$
C_{f f}(t)=\operatorname{tr}\left[\hat{F}(\beta / 2) e^{i \hat{H} t / \hbar} \hat{F}(\beta / 2) e^{-i \hat{H} t / \hbar}\right],
$$

where $\hat{F}(\beta / 2)$ is the half-Boltzmannized flux operator,

$$
\hat{F}(\beta / 2)=e^{-\beta \hat{H} / 4} \hat{F} e^{-\beta \hat{H} / 4},
$$

which, like $\hat{F}(\beta)$, is of low rank. ${ }^{16,20}$ The critical quantities required for the transition state theory are $C_{f f}(0)$ and $\ddot{C}_{f f}(0)$. Note that all the odd derivatives are zero since $C_{f f}(t)$ is an even function of time. [See the Appendix for a comparison of using Eqs. (1.2) and (1.3) vs Eqs. (2.4) and (2.5) for the current problem.]

Here we show how the low rank of $\hat{F}(\beta / 2)$ can be used to efficiently obtain $C_{f f}(0)$ and $\ddot{C}_{f f}(0)$. The first step is to obtain the eigenstates of the half-Boltzmannized flux operator

$$
\hat{F}(\beta / 2)|m\rangle=f_{m}|m\rangle
$$

with nonzero eigenvalues. This can be accomplished using an iterative Lanczos scheme. ${ }^{27,28}$ This basis of eigenstates can then be used to evaluate the trace required to obtain $C_{f f}(t) .{ }^{16,20}$ Then the flux-flux autocorrelation function at zero time is 


$$
\begin{aligned}
C_{f f}(0) & =\operatorname{tr}\{\hat{F}(\beta / 2) \hat{F}(\beta / 2)\}, \\
& =\sum_{m}\langle m|\hat{F}(\beta / 2) \hat{F}(\beta / 2)| m\rangle, \\
& =\sum_{m} f_{m}^{2} .
\end{aligned}
$$

The second derivative evaluated at $t=0$ can be straightforwardly calculated as

$$
\begin{aligned}
\ddot{C}_{f f}(0)= & \left(\frac{i}{\hbar}\right)^{2} \operatorname{tr}\{\hat{F}(\beta / 2)[\hat{H},[\hat{H}, \hat{F}(\beta / 2)]]\}, \\
= & -\frac{2}{\hbar^{2}} \sum_{m} f_{m}\left[f_{m}\left\langle m\left|\hat{H}^{2}\right| m\right\rangle\right. \\
& \left.-\sum_{m^{\prime}} f_{m^{\prime}}\left|\left\langle m^{\prime}|\hat{H}| m\right\rangle\right|^{2}\right] .
\end{aligned}
$$

It is clear from these expressions that eigenstates with $f_{m}$ $\simeq 0$ will not contribute as both $C_{f f}(0)$ and $\ddot{C}_{f f}(0)$ consist only of quantities proportional to $f_{m}^{2}$ or $f_{m} f_{m^{\prime}}$. It is also noteworthy that the only work required to obtain $C_{f f}(0)$ and $\ddot{C}_{f f}(0)$ once the eigenstates are known is a single multiplication of the Hamiltonian onto each eigenvector $(\hat{H}|m\rangle)$ and some vector products.

At this point it is useful to consider the computational savings realized in this approximate approach. In a fully rigorous calculation of $C_{f f}(t)$ to obtain the rate, each eigenstate of $\hat{F}(\beta / 2)$ must be propagated in real time up to $\tau / 2$, where $\tau$ is the time in which $C_{f f}(t)$ decays to zero. ${ }^{20}$ Conversely, Eqs. (2.7) and (2.8) require no time propagation, but only a single Hamiltonian multiplication on each eigenvector. Exact calculations have been carried out for several reactions involving three and four atoms, ${ }^{12,16-20}$ indicating that the transition state theory should be applicable to quite large systems.

We note that a general expression for the derivatives of the flux-flux autocorrelation function evaluated at $t=0$ can be found, giving the $K$ th derivative as

$$
C_{f f}^{(K)}(0)=\sum_{m, m^{\prime}} f_{m} f_{m^{\prime}} \sum_{k=0}^{K} P_{k}^{K}\left\langle m_{K-k} \mid m^{\prime}\right\rangle\left\langle m^{\prime} \mid m_{k}\right\rangle,
$$

where $\left|m_{k}\right\rangle=\hat{H}^{k}|m\rangle$ and

$$
P_{k}^{K}=(-1)^{k} \frac{K !}{k !(K-k) !} .
$$

(Note that no more than $K / 2$ multiplications of the Hamiltonian onto each eigenvector is required since for even $k$

$$
\left\langle m^{\prime} \mid m_{k}\right\rangle=\left\langle m_{k / 2}^{\prime} \mid m_{k / 2}\right\rangle,
$$

and an analogous, though less symmetrical, division can be made for odd $k$.)

\section{THE D+H $\mathrm{H}_{2}$ REACTION}

We now consider an application of the quantum transition state theory of Hansen and Andersen as described in

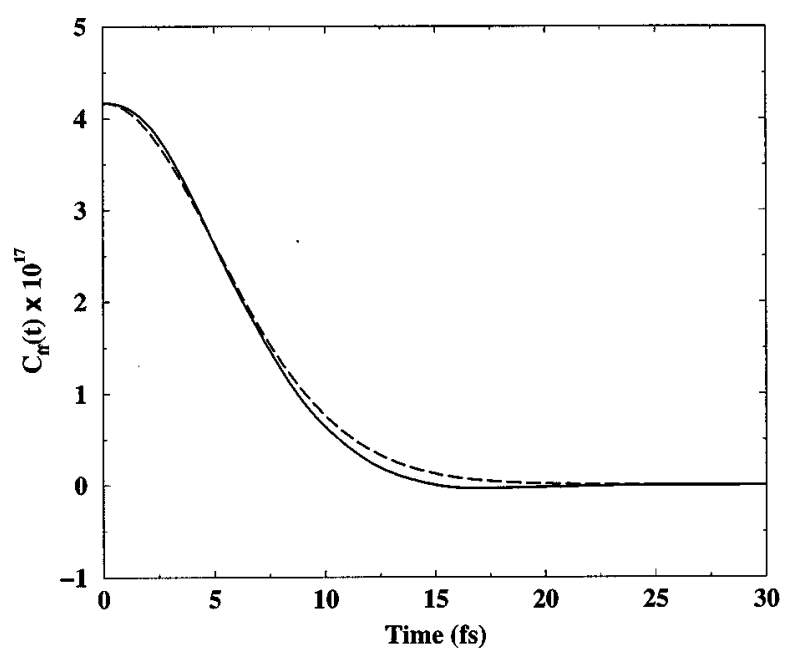

FIG. 1. Flux-flux autocorrelation function for the $\mathrm{D}+\mathrm{H}_{2}$ reaction at $T$ $=300 \mathrm{~K}$ for even parity. The quantum transition state theory result (dashed line) using Eq. (2.2) is compared with the exact correlation function (solid line). The units of the correlation function are (atomic units of time $)^{-2}$.

Sec. II in order to illustrate its utility for multidimensional systems. Specifically, we calculate the thermal rate constant for the $\mathrm{D}+\mathrm{H}_{2}$ reaction for zero total angular momentum $(J$ $=0$ ). This provides a useful test as the reaction is known to be direct and the quantum transition state theory is therefore expected to give accurate rates.

In this section we compare rate constants for the $\mathrm{D}+\mathrm{H}_{2}$ reaction obtained from the quantum transition state theory approach to the exact rate constants obtained by a full calculation of the flux-flux autocorrelation function. In this way, ambiguities arising from the use of different potential energy surfaces and/or theoretical approaches are eliminated and the approximation of $C_{f f}(t)$ is directly tested. The specifics of the computational approach for calculating the exact rate constant for the $\mathrm{D}+\mathrm{H}_{2}$ reaction has been given in detail elsewhere. ${ }^{29}$ The implementation is the same for the quantum transition state theory except for the approximations described in Sec. II. The approximate correlation function is taken to be of the form given in Eq. (2.2).

Figure 1 shows the flux-flux autocorrelation function for the $\mathrm{D}+\mathrm{H}_{2}$ reaction (for even parity) at $T=300 \mathrm{~K}$ obtained exactly and from the transition state theory approximation of Sec. II. The two correlation functions are in good agreement. Note that the approximate correlation function is not greater than the exact correlation function at all times, but for this temperature does yield a thermal rate constant larger than the exact result. The exact correlation function does become slightly negative around $15 \mathrm{fs}$, while the transition state theory correlation function decreases monotonically and remains positive at all times.

An Arrhenius plot for the $\mathrm{D}+\mathrm{H}_{2}$ reaction for total angular momentum $J=0$ is shown in Fig. 2 at $T=300-1500 \mathrm{~K}$. The rate constants obtained from an exact evaluation of the flux-flux correlation function are compared with those from the quantum transition state theory. For reference, the exact rate constants agree to within $2.5 \%$ with the previous exact calculations of Mielke et al. ${ }^{30}$ over this temperature 


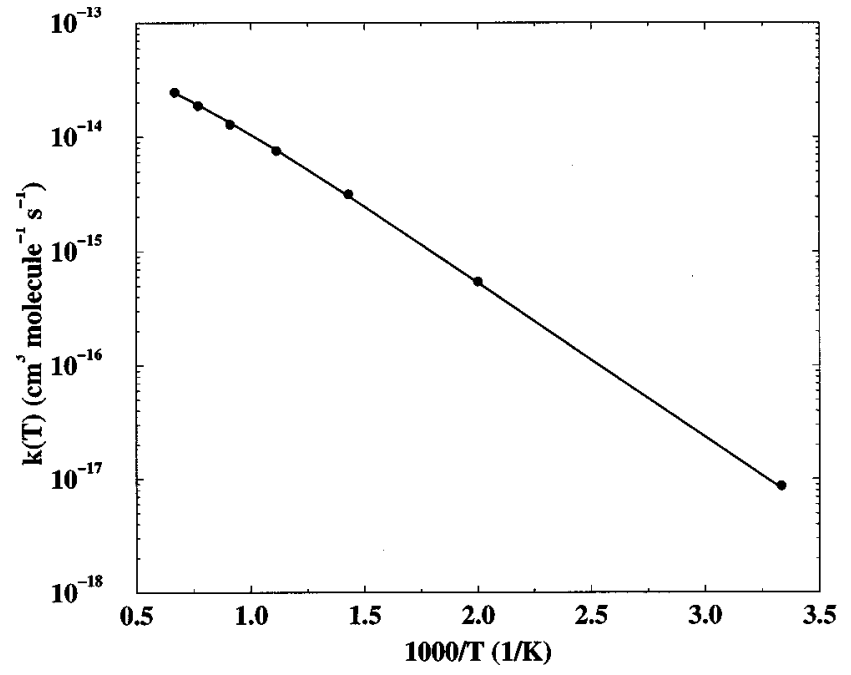

FIG. 2. Arrhenius plot for the $\mathrm{D}+\mathrm{H}_{2}$ reaction for zero total angular momentum $(J=0)$. The rate constants obtained from an exact evaluation of the flux-flux autocorrelation function (solid line) and the quantum transition state theory result (filled circles) are shown.

range. The agreement between the approximate and exact rates is excellent; the rates are within 5\% at all temperatures shown. It is interesting to note that the transition state theory rate constants are smaller than the exact results for $T \geqslant 900$ $\mathrm{K}$. It would be interesting to examine the variational nature of the quantum transition state theory by "optimizing', the flux dividing surface to minimize the rate constant. ${ }^{8,9}$

\section{SEPARABLE TRANSITION STATE THEORY}

It is instructive to examine the relation of the quantum mechanical transition state theory described in Sec. II to the "conventional" formulation. The conventional quantum transition state theory is given as the quantized version of the classical TST rate,

$$
k_{\mathrm{QM}}^{\mathrm{TST}}(T)=\Gamma(T) \frac{k_{b} T}{h} \frac{Q^{\ddagger}(T)}{Q_{r}(T)} e^{-\beta E_{b}},
$$

where $Q_{r}(T)$ and $Q^{\ddagger}(T)$ are the (quantum mechanical) partition functions for the reactants and the activated complex, respectively, $E_{b}$ is the barrier height, and $\Gamma(T)$ is a factor accounting for the effects of tunneling, the tunneling correction. Note that this formulation of quantum TST involves an assumption of separability between the reaction coordinate (i.e., the normal mode coordinate at the transition state with an imaginary frequency) and the remaining degrees of freedom at the transition state; $Q^{\ddagger}(T)$ is calculated in the degrees of freedom orthogonal to the reaction coordinate at the transition state. Typically $\Gamma(T)$ is a one-dimensional tunneling correction factor, though it sometimes includes some effect of the curvature of the reaction path. ${ }^{4}$

Now consider the thermal rate constant as obtained from the flux-flux autocorrelation function. If we assume separability between the reaction coordinate, which we denote by $s$, and the bath at the transition state such that the Hamiltonian can be written as

$$
\hat{H}=\hat{H}_{s}+\hat{H}_{b}\left(s=s_{0}\right),
$$

where $s=s_{0}$ defines the flux dividing surface then

$$
\begin{aligned}
k(T) & =\frac{1}{Q_{r}(T)} \int_{0}^{\infty} C_{f f}(t) d t, \\
& =\frac{1}{Q_{r}(T)} \operatorname{tr}_{b}\left[e^{-\beta H_{b}\left(s_{0}\right)}\right] \int_{0}^{\infty} C_{f f}^{s}(t) d t, \\
& =\frac{Q^{\ddagger}(T)}{Q_{r}(T)} \int_{0}^{\infty} C_{f f}^{s}(t) d t .
\end{aligned}
$$

We have used the fact that $\hat{H}_{b}\left(s_{0}\right)$ commutes with $\hat{H}_{s}$ and $\hat{F}$, and

$$
Q^{\ddagger}(T)=\operatorname{tr}_{b}\left[e^{-\beta H_{b}\left(s_{0}\right)}\right] .
$$

The flux-flux autocorrelation function $C_{f f}^{s}(t)$ is that for the reaction coordinate alone, i.e., Eq. (1.2) [or Eq. (2.4)] with the full Hamiltonian replaced by $\hat{H}_{s}$. Equation (4.3) can be written in the form of Eq. (4.1) with the tunneling correction given by

$$
\Gamma(T)=\frac{h}{k_{b} T} e^{\beta E_{b}} \int_{0}^{\infty} C_{f f}^{s}(t) d t .
$$

It is worth noting that for the free particle (with $E_{b}=0$ ), for which the correlation function is

$$
C_{f f}^{f p}(t)=\frac{k_{b} T}{h} \frac{(\hbar \beta / 2)^{2}}{\left[t^{2}+(\hbar \beta / 2)^{2}\right]^{3 / 2}},
$$

one obtains $\Gamma(T)=1$. For the case of a parabolic barrier, with $C_{f f}(t)$ given by Eq. (2.1),

$$
\Gamma(T)=\frac{\hbar \beta \omega_{b} / 2}{\sin \left(\hbar \beta \omega_{b} / 2\right)},
$$

which is the exact result previously obtained by Miller. ${ }^{3}$ (The standard Wigner tunneling correction ${ }^{31}$ is the expansion of this expression to lowest order in $\hbar$.) Naturally, the quantum TST of Hansen and Andersen obtains the correct result for the case of the parabolic barrier using Eq. (2.1).

The separable quantum transition state theory given by Eq. (4.3) may be calculated with the exact $C_{f f}^{s}(t)$ or with $C_{f f}^{s}(t)$ replaced by its approximate form within the TST of Hansen and Andersen. It is important to note that Eq. (4.5) cannot always be applied using the exact correlation function since the rate in the reaction coordinate alone is not always well-defined. For example, the flux-flux autocorrelation function for the one-dimensional double well potential considered in Sec. V oscillates indefinitely and thus there is not a well defined rate for the reaction coordinate alone. (However, in such cases the quantum TST of Hansen and Andersen can still provide a useful evaluation of the rate, as will be seen shortly.) When the double well is coupled to a harmonic bath, which is the system one wishes to describe, there can be a well defined rate as the bath can withdraw energy from the reaction coordinate. The system can thereby relax into the product well, eliminating repeated recrossing of the transition state.

The assumption of separability at the transition state made in Eq. (4.2) could equally well be carried out while including several coordinates in $\hat{H}_{s}$. The separability ap- 
proximation can thereby be improved since all the coordinates in $\hat{H}_{s}$ are treated fully coupled in the quantum transition state theory of Hansen and Andersen.

\section{DOUBLE WELL COUPLED TO A HARMONIC BATH}

\section{A. Description of the problem}

To illustrate the quantum mechanical transition state theory we apply it to the problem of a symmetric double well potential bilinearly coupled to a harmonic bath. This problem has been studied in great detail by a large number of workers $^{32,33}$ and, importantly for the present purpose, exact calculations for the rate constant have been carried out by Topaler and $\mathrm{Makri}^{24}$ using the quasiadiabatic propagator path integral approach with an influence functional. The Hamiltonian can be written as

$$
\begin{aligned}
\hat{H}= & \frac{\hat{p}_{x}^{2}}{2 \mu}-\frac{1}{2} \mu \omega_{b}^{2} \hat{x}^{2}+\frac{\mu^{2} \omega_{b}^{4}}{16 E_{b}} \hat{x}^{4} \\
& +\sum_{j=1}^{N}\left[\frac{\hat{p}_{j}^{2}}{2 m_{j}}+\frac{1}{2} m_{j} \omega_{j}^{2} \hat{q}_{j}^{2}\right] \\
& -\sum_{j=1}^{N} c_{j} \hat{q}_{j} \hat{x}+\sum_{j=1}^{N} \frac{c_{j}^{2}}{2 m_{j} \omega_{j}^{2}} \hat{x}^{2},
\end{aligned}
$$

where $\omega_{b}$ is the barrier frequency and the $\omega_{j}$ are the frequencies of the bath. The last term is a renormalization factor which ensures that the barrier height, $E_{b}$, remains the same as the system-bath interaction, defined by the coupling constants $\left\{c_{j}\right\}$, changes. We consider the parameter values in the "DW1" potential of Topaler and Makri ${ }^{24}$ with $\omega_{b}=500$ $\mathrm{cm}^{-1}, E_{b}=2085 \mathrm{~cm}^{-1}$, and $\mu=1837.15$ a.u. (the mass of a hydrogen atom).

The characteristics of the bath are encompassed in the spectral density, $J(\omega)$, via the relation ${ }^{34}$

$$
J(\omega)=\frac{\pi}{2} \sum_{j} \frac{c_{j}^{2}}{m_{j} \omega_{j}} \delta\left(\omega-\omega_{j}\right) .
$$

Here we assume an Ohmic spectral density with an exponential cutoff,

$$
J(\omega)=\eta \omega e^{-\omega / \omega_{c}},
$$

where $\eta$ is a measure of the system-bath interaction and is related to the friction of the harmonic bath and $\omega_{c}$ is the cutoff frequency (taken to be $500 \mathrm{~cm}^{-1}$ ). We represent the bath as a set of (300) oscillators with equally spaced discrete frequencies with a maximum frequency of $5 \omega_{c}$. Then, the coupling constant for a given oscillator is given by the relation

$$
\frac{c_{j}^{2}}{m_{j} \omega_{j}}=\frac{2}{\pi} \eta \omega_{j} e^{-\omega / \omega_{c} \Delta \omega,}
$$

where $\Delta \omega$ is the frequency spacing.

The reaction coordinate is obtained by solving for the normal mode coordinates of the potential in Eq. (5.1) and is given by the mode with an imaginary frequency. The bath modes are then the remaining normal mode coordinates. We approximate the reaction coordinate potential as a symmetric double well such that

$$
\hat{H}_{s}=\frac{\hat{p}_{s}^{2}}{2}-\frac{1}{2} \omega_{\ddagger}^{2} \hat{s}^{2}+\frac{\omega_{\ddagger}^{4}}{16 E_{b}} \hat{s}^{4},
$$

where $\omega_{\ddagger}$ is the absolute value of the imaginary normal mode frequency of the (mass-weighted) reaction coordinate $s$. The bath Hamiltonian is given by

$$
\hat{H}_{b}=\sum_{j=1}^{N}\left[\frac{\hat{P}_{j}^{2}}{2}+\frac{1}{2} \Omega_{j}^{2} \hat{Q}_{j}^{2}\right],
$$

where the $\Omega_{j}$ are the bath mode frequencies corresponding to the (mass-weighted) coordinates $Q_{j}$, obtained from the normal mode analysis at the transition state. In defining the reaction coordinate to be the imaginary frequency normal mode coordinate we are, in effect, making a choice of the flux dividing surface designed to improve the accuracy of the transition state theory. We have not carried out an explicit optimization of the dividing surface, as suggested by Hansen and Andersen, ${ }^{8,9}$ which may improve the accuracy of the rate constants.

The classical Hamiltonian corresponding to Eq. (5.1), with a system coordinate bilinearly coupled to a harmonic bath, is equivalent to the system coordinate obeying a generalized Langevin equation. ${ }^{35}$ Grote and Hynes have obtained a simple and elegant expression for the rate constant for this problem, using a parabolic approximation to the barrier. ${ }^{36}$ Pollak has shown that their approach is equivalent to classical transition state theory applied in the normal mode coordinates of the transition state. ${ }^{37}$ Quantum mechanical effects can be included in the Grote-Hynes theory rate constants by a correction factor derived by Wolynes. ${ }^{38}$

Finally, we note some computational details. In calculating $C_{f f}(0)$ and $\ddot{C}_{f f}(0)$ by Eqs. (2.7) and (2.8), respectively, a sinc-function discrete variable representation basis ${ }^{39}$ is used for the one-dimensional double well potential. The flux operator is used in the commutator form,

$$
\hat{F}=\frac{i}{\hbar}\left[\hat{H}_{s}, h(s)\right] .
$$

Four Lanczos iterations are performed to obtain the nonzero eigenvalues of $\hat{F}(\beta / 2)$ in Eq. (2.5) and their corresponding eigenvalues. The ratio of partition functions, $Q^{\ddagger}(T) / Q_{r}(T)$, in Eq. (4.3) is obtained using a normal mode analysis for both the reactants and the activated complex.

\section{B. Results and discussion}

Here we present the results for the double well bilinearly coupled to a harmonic bath using the method described in Secs. II and IV.

Following Topaler and Makri, ${ }^{24}$ we report the rates for the double well potential bilinearly coupled to a harmonic bath as the transmission coefficient

$$
\kappa(T)=\frac{k(T)}{k_{\mathrm{TST}}(T)} .
$$




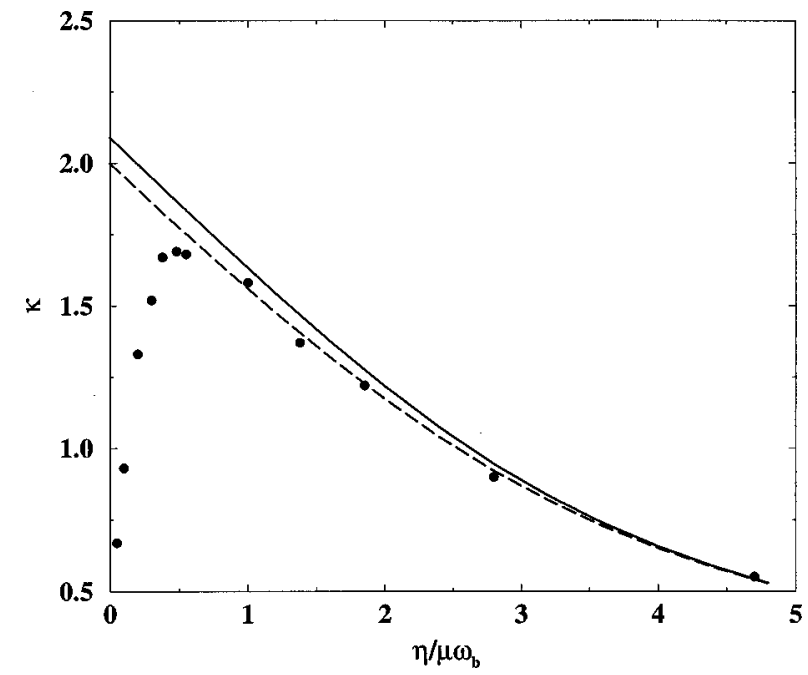

FIG. 3. Transmission coefficient for the double well potential bilinearly coupled to a harmonic bath at $T=300 \mathrm{~K}$ vs the coupling strength parameter $\eta /\left(\mu \omega_{b}\right)$. Results are shown for the present method as given in Eq. (4.3), (solid line), the parabolic barrier tunneling correction, (dashed line), and the exact results of Topaler and Makri (Ref. 24), (solid circles).

The primitive transition state theory rate, $k_{\mathrm{TST}}(T)$, is given by

$$
k_{\mathrm{TST}}(T)=\frac{\omega_{0}}{2 \pi} e^{-\beta E_{b}},
$$

where $\omega_{0}$ is the frequency in the reactant well $\left(\omega_{0}=707\right.$ $\mathrm{cm}^{-1}$ for the parameters used here).

Transmission coefficients are presented for the approach described in Sec. IV, specifically the rate given by Eq. (4.3). The correlation function for the reaction coordinate alone, $C_{f f}^{s}(t)$ is obtained using the Padé approximant form of Hansen and Andersen, ${ }^{9}$ Eq. (2.2), for the correlation function. The values of $C_{f f}(0)$ and $\ddot{C}_{f f}(0)$ are obtained from Eqs. (2.7) and (2.8), respectively and used to determine the parameters $a$ and $b$ via Eqs. (2.3). For comparison we have carried out calculations using the parabolic tunneling correction from Eq. (4.1) using Eq. (4.7) for $\Gamma(T)$.

Figure 3 shows the transmission coefficient at $T=300 \mathrm{~K}$ vs the parameter $\eta /\left(\mu \omega_{b}\right)$ governing the coupling strength [see Eqs. (5.3) and (5.4)]. The exact calculations of Topaler and $\mathrm{Makri}^{24}$ and the results obtained using the traditional parabolic barrier tunneling correction are plotted for comparison. It is immediately obvious that the transition state theory (with either tunneling correction) does not reproduce the correct behavior of the transmission coefficient for small coupling. This regime is dominated by recrossing effects as the small coupling inhibits the relaxation of the system in the product well resulting in repeated recrossing of the transition state. Since the transition state theory makes no accounting for recrossing dynamics, it severely overestimates the rate for small coupling. However, for intermediate to large coupling $\left[\eta /\left(\mu \omega_{b}\right)\right.$ greater than $\left.\sim 1\right]$, the transition state theory correctly (and quantitatively) predicts the decrease of the rate with increased coupling. This is because the dynamics for this range of coupling strength is direct, i.e., it is characterized by little recrossing of the transition state. The transmis-

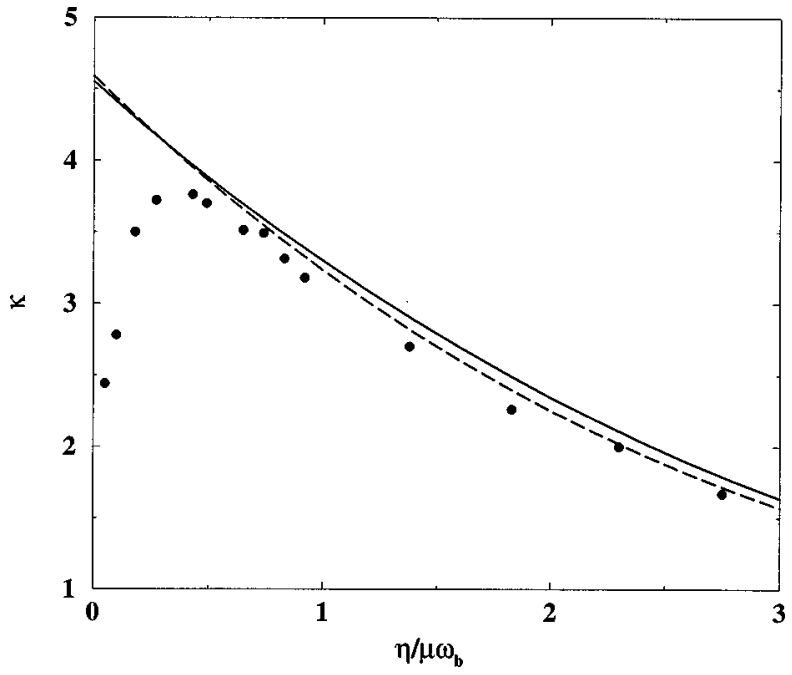

FIG. 4. Same as Fig. 3 but for $T=200 \mathrm{~K}$.

sion coefficient obtained from Eq. (4.3) is slightly larger than that obtained from the parabolic barrier tunneling correction, by less that $5 \%$.

The transmission coefficient is plotted vs the coupling strength parameter $\eta /\left(\mu \omega_{b}\right)$ at a lower temperature, $T$ $=200 \mathrm{~K}$, in Fig. 4. Again the transition state theory is significantly in error for small coupling but reproduces the transmission coefficient for larger coupling $\left[\eta /\left(\mu \omega_{b}\right)\right.$ greater than $\sim 0.5]$ quite well. The present method and the parabolic tunneling correction are in very good agreement over the entire range of coupling strength.

Finally, Fig. 5 plots the logarithm of the transmission coefficient as a function of $\eta /\left(\mu \omega_{b}\right)$ at $T=100 \mathrm{~K}$. Note that at this temperature the exact transmission coefficient exhibits a turnover (such as those observed in Figs. 3 and 4) at exponentially small coupling ${ }^{24}$ and so it does not appear in this data. At this low temperature the parabolic tunneling correction does not give a useful rate for small coupling [where $T$ is below the crossover temperature, i.e., defined as that for which Eq. (4.7) diverges, given by $\left.T_{c}=\hbar \omega_{\ddagger} /\left(2 \pi k_{b}\right)\right]$. How-

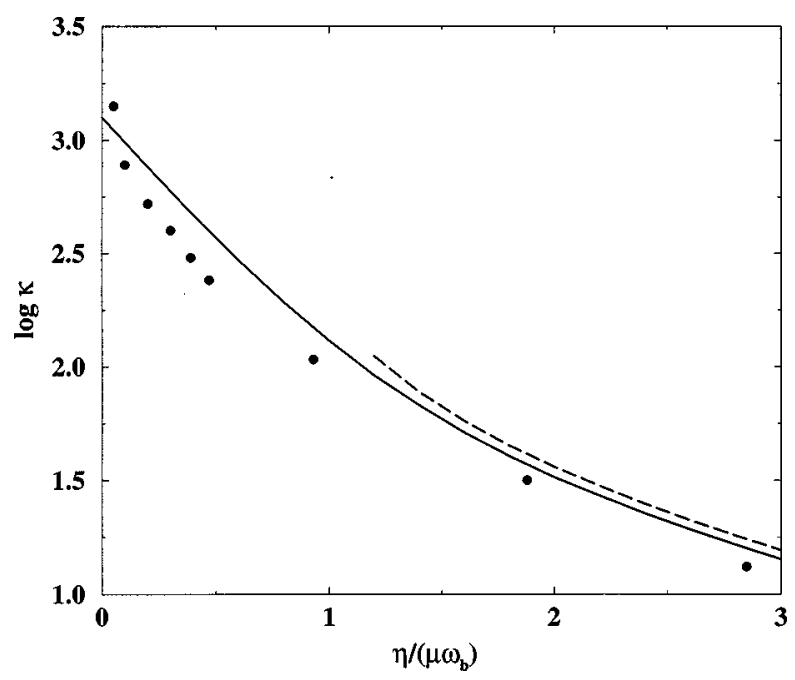

FIG. 5. Same as Fig. 3 but for $T=100 \mathrm{~K}$ and here the logarithm of the transmission coefficient is plotted. 
ever, because the present approach for obtaining the tunneling correction is based on a physically realistic onedimensional potential it does give meaningful rates in this regime. The present transition state theory approach gives transmission coefficients in reasonable agreement with the exact calculation over the entire range of coupling. The results from the parabolic barrier tunneling correction are shown for larger values of the coupling and are in good agreement with the present method.

It is instructive to consider how the present method compares to other quantum transition state theory approaches. Topaler and Makri ${ }^{24}$ compared their exact results with those from Grote-Hynes theory ${ }^{36}$ with a quantum correction ${ }^{38}$ and centroid density theory. ${ }^{6}$ At $T=300$ and $200 \mathrm{~K}$, these approaches significantly overestimate the rate constants for small coupling, as does the present method, but are in excellent agreement with the exact results for larger coupling. ${ }^{24}$ The centroid density theory gives rates in good agreement with the exact results at $T=100 \mathrm{~K}$, while Grote-Hynes theory is in poor agreement for small coupling and reasonable agreement above $\eta /\left(\mu \omega_{b}\right) \sim 1.5$. Thus, the present method is capable of obtaining rate constants of accuracy comparable to either of these approaches.

\section{CONCLUDING REMARKS}

We have shown how the quantum transition state theory recently proposed by Hansen and Andersen ${ }^{8,9}$ can be efficiently implemented by taking advantage of the low rank of the half-Boltzmannized flux operator. This approach can be easily applied to systems with several degrees of freedom. We have also described how the method of Hansen and Andersen can be used to obtain accurate tunneling corrections within the context of the more traditional (i.e., separable) quantized transition state theory approach. An important addendum is that the present approach can be used to improve the separability approximation in such TSTs by explicitly treating multiple (fully coupled) degrees of freedom in the calculation of the tunneling correction.

We have demonstrated the present implementation the quantum transition state theory of Hansen and Andersen by using it to calculate thermal rate constants for the $\mathrm{D}+\mathrm{H}_{2}$ reaction. This reaction is known to be direct and the quantum transition state theory gives rate constants in excellent agreement with exact results.

We have also used the present method to calculate tunneling corrections for a one-dimensional double well potential bilinearly coupled to a harmonic bath. This transition state theory approach severely overestimates the transmission coefficient, Eq. (5.8), when there is significant recrossing of the transition state (as would be expected). However, when the transition state theory ansatz of direct dynamics is met the present results are in good agreement (at $T$ $=100,200$, and $300 \mathrm{~K}$ ) with the exact results obtained by Topaler and Makri. ${ }^{24}$ The use of a physically realistic potential for obtaining the tunneling correction allows for an accurate rate to be obtained even at temperatures below the crossover temperature (where, for example, the parabolic barrier tunneling correction is not valid).
Finally, we offer some suggestions for possible improvements to the theory. While the Padé approximant form for the correlation function, Eq. (2.2), proposed by Hansen and Andersen possesses many desirable characteristics it is possible to obtain a positive value for the parameter $b$ given by Eq. (2.3b), resulting in a meaningless value for the rate. (We observe this for the double well problem in Sec. $\mathrm{V}$ for $T$ $\simeq 50 \mathrm{~K}$.) Thus, a form for the correlation function which always yields a useful rate (while still possessing the other desired properties) is wanting.

Another possibility for improvement involves moving beyond the separability approximation of Sec. IV. It should be possible to include some effects of the coupling in the calculation of $C_{f f}(0)$ and $\ddot{C}_{f f}(0)$ by a perturbative or otherwise approximate approach.

As shown in Sec. II and the Appendix it is possible to calculate many derivatives of the flux-flux autocorrelation function (evaluated at $t=0$ ), via Eq. (2.9), and it should be possible to use this additional information to obtain more accurate representations of the correlation function, including the description of recrossing dynamics. A natural extension is to approximate the correlation function using a functional form with more parameters, and thus requiring the values of higher derivatives at $t=0$. Naturally, such an approach involves moving beyond the traditional assumption of a "transition state theory," namely, that of direct dynamics. More systematic approaches have previously been applied in different contexts. ${ }^{40,41}$

\section{ACKNOWLEDGMENTS}

It is a pleasure to thank Professor William H. Miller for his encouragement as well as for many fruitful discussions and useful suggestions. I also wish to acknowledge Dr. Haobin Wang for several productive conversations and helpful comments. I am grateful to Professor James T. Hynes for his generous support.

\section{APPENDIX: TIME DERIVATIVES OF THE FLUX-FLUX AUTOCORRELATION FUNCTION}

In this Appendix we derive a general expression for the $K$ th derivative of the flux-flux autocorrelation function evaluated at zero time. In particular, we exploit the low rank of the Boltzmannized flux operator,

$$
\hat{F}(\beta)=e^{-\beta \hat{H} / 2} \hat{F} e^{-\beta \hat{H} / 2},
$$

which appears in Eq. (1.2) for the correlation function. Thus, if the nonzero eigenvalues and eigenvectors of $\hat{F}(\beta)$ are obtained by a Lanczos scheme

$$
\hat{F}(\beta)|n\rangle=f_{n}|n\rangle,
$$

then the correlation function becomes

$$
C_{f f}(t)=\sum_{n} f_{n}\left\langle n\left|e^{i \hat{H} t / \hbar} \hat{F} e^{-i \hat{H} t / \hbar}\right| n\right\rangle
$$

It is easy to show that the time derivatives of $C_{f f}(t)$ evaluated at zero time are given by expressions containing com- 
mutators between the Hamiltonian and the flux operator. For the first two nonzero derivatives, one obtains

$$
C_{f f}(0)=\sum_{n} f_{n}\langle n|\hat{F}| n\rangle
$$

and

$$
\ddot{C}_{f f}(0)=C_{f f}^{(2)}(0)=\left(\frac{i}{\hbar}\right)^{2} \sum_{n} f_{n}\langle n|[\hat{H},[\hat{H}, \hat{F}]]| n\rangle .
$$

Then the derivative $C_{f f}^{(K)}(0)$ is composed of $K$ nested commutators of the Hamiltonian with the flux operator. However, the $K$ th nested commutator can be expressed as

$$
\begin{aligned}
& P_{K}^{K} \hat{H}^{K} \hat{F}+P_{K-1}^{K} \hat{H}^{K-1} \hat{F} \hat{H}+P_{K-2}^{K} \hat{H}^{K-2} \hat{F} \hat{H}^{2}+\cdots \\
& \quad+P_{1}^{K} \hat{H} \hat{F} \hat{H}^{K-1}+P_{0}^{K} \hat{F} \hat{H}^{K},
\end{aligned}
$$

where the coefficients $P_{K-k}^{K}$ are those given in Eq. (2.10). It is not hard to see that if we apply powers of the Hamiltonian to the eigenvectors of the Boltzmannized flux operator to generate the vectors $\left|n_{k}\right\rangle=\hat{H}^{k}|n\rangle$ that the $K$ th derivative is given by

$$
C_{f f}^{(K)}(0)=\left(\frac{i}{\hbar}\right)^{K} \sum_{n} f_{n} \sum_{k=0}^{K} P_{k}^{K}\left\langle n_{K-k}|\hat{F}| n_{k}\right\rangle,
$$

which is the equivalent of Eq. (2.9).

The method of calculating the derivatives utilizing the half-Boltzmannized flux operator presented in Sec. II presents some advantages over that given here. A shorter propagation in imaginary time $\left(t_{c}=-i \beta \hbar / 4\right.$ rather than $-i \beta \hbar / 2)$ is required and fewer multiplications of the Hamiltonian onto the eigenvectors are needed.

${ }^{1}$ H. Eyring, J. Chem. Phys. 3, 107 (1935); Trans. Faraday Soc. 34, 41 (1938).

${ }^{2}$ E. Wigner, Trans. Faraday Soc. 34, 29 (1938).

${ }^{3}$ W. H. Miller, J. Chem. Phys. 61, 1823 (1974).

${ }^{4}$ For a review of conventional transition state theory approaches and quantum corrections, see D. G. Truhlar, in The Theory of Chemical Reaction Dynamics, edited by M. Baer (Chemical Rubber, Boca Raton, 1985), Vol. IV, Chap. 2.

${ }^{5}$ J. W. Tromp and W. H. Miller, J. Phys. Chem. 90, 3482 (1986).

${ }^{6}$ G. A. Voth, D. Chandler, and W. H. Miller, J. Chem. Phys. 91, 7749 (1989); G. A. Voth, Chem. Phys. Lett. 170, 289 (1990).

${ }^{7}$ D. G. Truhlar and B. C. Garrett, J. Phys. Chem. 96, 6515 (1992).
${ }^{8}$ N. F. Hansen and H. C. Andersen, J. Chem. Phys. 101, 6032 (1994).

${ }^{9}$ N. F. Hansen and H. C. Andersen, J. Phys. Chem. 100, 1137 (1996).

${ }^{10}$ T. Yamamoto, J. Chem. Phys. 33, 281 (1960).

${ }^{11}$ W. H. Miller, S. D. Schwartz, and J. W. Tromp, J. Chem. Phys. 79, 4889 (1983).

${ }^{12}$ W. H. Thompson and W. H. Miller, J. Chem. Phys. 106, 142 (1997); 107, 2164(E) (1997); H. Wang, W.H. Thompson, and W. H. Miller, ibid. 107, 7194 (1997); W. H. Miller, J. Phys. Chem. A 102, 793 (1998).

${ }^{13}$ T. J. Park and J. C. Light, J. Chem. Phys. 85, 5870 (1986).

${ }^{14}$ T. J. Park and J. C. Light, J. Chem. Phys. 88, 4897 (1988).

${ }^{15}$ T. Seideman and W. H. Miller, J. Chem. Phys. 95, 1768 (1991).

${ }^{16} \mathrm{H}$. Wang, W. H. Thompson, and W. H. Miller, J. Phys. Chem. A 102, $9372(1998)$

${ }^{17}$ W. H. Thompson and W. H. Miller, J. Chem. Phys. 102, 7409 (1995).

${ }^{18}$ T. C. Germann and W. H. Miller, J. Phys. Chem. A 101, 6358 (1997); D. E. Skinner, T. C. Germann, and W. H. Miller, ibid. 102, 3828 (1998).

${ }^{19}$ U. Manthe, J. Chem. Phys. 102, 9205 (1995); U. Manthe and F. Matzkies, Chem. Phys. Lett. 252, 71 (1996)

${ }^{20}$ F. Matzkies and U. Manthe, J. Chem. Phys. 106, 2646 (1997); U. Manthe and F. Matzkies, Chem. Phys. Lett. 282, 442 (1998); F. Matzkies and U. Manthe, J. Chem. Phys. 108, 4828 (1998).

${ }^{21}$ W. H. Miller, J. Phys. Chem. 99, 12387 (1995); Faraday Discuss. 102, 53 (1995).

${ }^{22}$ D. Brown and J. C. Light, J. Chem. Phys. 97, 5465 (1992).

${ }^{23}$ D. H. Zhang and J. C. Light, J. Chem. Phys. 104, 6184 (1996); 106, 551 (1997); D. H. Zhang, J. C. Light, and S.-Y. Lee, ibid. 109, 79 (1998).

${ }^{24}$ M. Topaler and N. Makri, J. Chem. Phys. 101, 7500 (1994).

${ }^{25}$ This functional form has previously been used in an analytic continuation approach to obtain $C_{f f}(t)$; see J. W. Tromp, Ph.D. thesis, Lawrence Berkeley Laboratory, University of California, 1988.

${ }^{26}$ J. Costley and P. Pechukas, Chem. Phys. Lett. 83, 139 (1981).

${ }^{27}$ C. Lanczos, J. Res. Natl. Bur. Stand. 45, 255 (1950).

${ }^{28}$ Y. Saad, Numerical Methods for Large Eigenvalue Problems (Halstead, New York, 1992)

${ }^{29}$ W. H. Thompson, in Highly Excited Molecules: Relaxation, Reaction and Structure, ACS Symposium Series 678, edited by A. S. Mullin and G. C. Schatz (American Chemical Society, Washington, D.C., 1997).

${ }^{30}$ S. L. Mielke, G. C. Lynch, D. G. Truhlar, and D. W. Schenke, J. Phys. Chem. 98, 7994 (1994).

${ }^{31}$ E. Wigner, Z. Phys. Chem. Abt. B 19, 203 (1932).

${ }^{32}$ For a review of the history of this problem the reader is referred to Refs. 24 and 34 and the references within.

${ }^{33}$ H. Wang, X. Sun, and W. H. Miller, J. Chem. Phys. 108, 9726 (1998).

${ }^{34}$ A. J. Leggett, S. Chakravarty, A. T. Dorsey, M. P. Fisher, A. Garg, and W. Zwerger, Rev. Mod. Phys. 59, 1 (1987).

${ }^{35}$ R. Zwanzig, J. Stat. Phys. 9, 215 (1973).

${ }^{36}$ R. F. Grote and J. T. Hynes, J. Chem. Phys. 73, 2715 (1980).

${ }^{37}$ E. Pollak, J. Chem. Phys. 85, 865 (1986).

${ }^{38}$ P. G. Wolynes, Phys. Rev. Lett. 47, 968 (1981).

${ }^{39}$ D. T. Colbert and W. H. Miller, J. Chem. Phys. 96, 1982 (1992).

${ }^{40}$ O. Platz and R. G. Gordon, Phys. Rev. Lett. 30, 264 (1973); Phys. Rev. B 7, 4764 (1973).

${ }^{41}$ J. C. Wheeler, Phys. Rev. A 9, 825 (1974). 